\title{
Electroweak and radiative penguin processes in B-decays at Belle
}

\author{
Luis PESÁNTEZ ${ }^{* \dagger}$ \\ University of Bonn \\ E-mail: pesantez@physik.uni-bonn.de
}

The transitions $b \rightarrow d \gamma, b \rightarrow s \gamma$ and $b \rightarrow s \ell^{+} \ell^{-}$are flavor-changing neutral currents, forbidden at tree level in the Standard Model (SM). These decays proceed via loop or box diagrams and can provide tests of new-physics effects. They provide a rich set of observables, that can be used to test the SM. The SM gives very precise predictions when the decays are considered inclusively, for this reason it is important to perform studies where as many final states as possible are reconstructed or where the decay is considered fully inclusively, without explicitly reconstructing the $B$ meson. The large Belle data set of $711 \mathrm{fb}^{-1}$ recorded at the $\Upsilon(4 S)$ resonance allows for precise measurements of radiative $B$-decays.

XXII. International Workshop on Deep-Inelastic Scattering and Related Subjects,

28 April - 2 May 2014

Warsaw, Poland

* Speaker.

${ }^{\dagger}$ on behalf of the Belle Collaboration 


\section{Introduction}

The decays $b \rightarrow d \gamma, b \rightarrow s \gamma$ and $b \rightarrow s \ell^{+} \ell^{-}$are flavor-changing neutral currents, which are forbidden at tree level in the Standard Model (SM). They proceed via loop or box diagrams making them sensitive to potential contributions of heavy non-SM particles. The effective Hamiltonian that governs weak decays can be expressed in the operator product expansion (OPE) [1] as the sum of operators $Q_{i}$ with corresponding Wilson coefficients $C_{i}: H_{\mathrm{eff}}=\frac{G_{F}}{\sqrt{2}} \sum_{i} V_{\mathrm{CKM}}^{i} C_{i}(\mu) Q_{i}$. Non-SM contributions would modify the predicted values of the Wilson coefficients.

The Belle detector at the KEKB $B$-factory is used to study $B$-mesons produced in the reaction $e^{+} e^{-} \rightarrow \Upsilon(4 S) \rightarrow B \bar{B}$. All studies presented here are performed with the complete Belle data set of $711 \mathrm{fb}^{-1}$. Some experimental techniques and variables are common to the following analyses. The consistency of a reconstructed $B$ meson with a $B$ decay can be checked using two variables: $M_{\mathrm{bc}}$ and $\Delta E$. They are defined as: $M_{\mathrm{bc}}=\sqrt{E_{\text {beam }}^{* 2}-p_{B}^{* 2}}$ and $\Delta E=E_{\mathrm{B}}^{*}-E_{\text {beam }}^{*}$, where $E_{B}^{*}$ and $p_{B}^{*}$ are the energy and momentum of the $B$ and $E_{\text {beam }}^{*}$ the energy of the beam, in the center-of-mass $(\mathrm{CM})$ frame. Continuum processes $\left(e^{+} e^{-} \rightarrow q \bar{q}, q=u, d, s, c\right)$ are an important background source. These events can be discriminated from $B \bar{B}$ since their decay products have a characteristic "jetlike" topology, different from $B \bar{B}$ events. Topological variables such as thrust and sphericity are used for continuum suppression. Additionally, Belle uses modified Fox-Wolfram moments [2]. Multivariate analysis techniques such as boosted decision trees (BDT), neural networks (NN) or likelihood ratios are employed to enhance the discriminating power.

\section{2. $C P$ asymmetry in $B \rightarrow X_{s+d} \gamma$}

The SM predicts non-vanishing $C P$ asymmetries $\left(\mathscr{A}_{C P}\right)$ for $B \rightarrow X_{d} \gamma$ and $B \rightarrow X_{s} \gamma$ decays [3]. However, when both decays are considered inclusively, the $C P$-violating contributions cancel due to CKM unitarity, and the theory errors cancel almost perfectly up to small corrections [4]. This $\mathrm{SM}$ prediction can serve as a clean test for new $C P$-violating phases. The asymmetry is defined as

$$
\mathscr{A}_{C P}=\frac{\Gamma(\bar{B} \rightarrow \bar{f})-\Gamma(B \rightarrow f)}{\Gamma(\bar{B} \rightarrow \bar{f})+\Gamma(B \rightarrow f)}
$$

with $\Gamma(B \rightarrow f)$ being the decay rate into the final state $f=X_{s+d} \gamma$.

To study the decay inclusively, only a photon is reconstructed from the decay of the signal $B$ meson with $\mathrm{CM}$ energy $E_{\gamma}^{*}=[1.7,2.8] \mathrm{GeV}$. The flavor of the signal $B$ is determined by tagging the other $B$ meson in the event by selecting a charged lepton originating from it. The charge of the lepton determines the flavor of the $B$ mesons. Photons from $\pi^{0}(\eta) \rightarrow \gamma \gamma$ decays are vetoed. The continuum background is suppressed using a BDT. A possible charge asymmetry in the detection of leptons is measured with a "tag-and-probe" approach in a $B \rightarrow X J / \psi\left(\ell^{+} \ell^{-}\right)$sample, we obtained $\mathscr{A}_{\text {det }}=(0.10 \pm 0.22) \times 10^{-2}$. A possible asymmetry in the $B \bar{B}$ background is measured in the sideband $E_{\gamma}^{*}=[1.4,1.7] \mathrm{GeV}$ yielding $\mathscr{A}_{\mathrm{bkg}}=(-0.14 \pm 0.78) \times 10^{-2}$. A wrong-tag factor $\omega$ appears in cases when the lepton charge gives the wrong signal $B$ flavor. This can be due to neutral $B$ oscillation, secondary leptons and hadrons misreconstructed as leptons. The measured and true asymmetries are related as: $\mathscr{A}_{C P}=\frac{1}{1-2 \omega} \mathscr{A}_{C P}^{\text {meas }}$, with $\omega=0.1413 \pm 0.0052$. 
The $B \rightarrow X_{s+d} \gamma$ spectrum after background subtraction and the asymmetry measurement for different photon energy thresholds in the range $E_{\gamma}^{*}=[1.7,2.2] \mathrm{GeV}$ are shown in Fig. 1. The asymmetry $\mathscr{A}_{C P}$ shows a stable behavior for the different energy thresholds and is consistent with the SM prediction. The measurement is statistically limited. For $E_{\gamma}^{*}>2.1 \mathrm{GeV}$ we find $\mathscr{A}_{C P}=$ $(2.2 \pm 4.0 \pm 0.8) \times 10^{-2}$. This measurement of $\mathscr{A}_{C P}\left(B \rightarrow X_{s+d} \gamma\right)$ is the most precise to date.
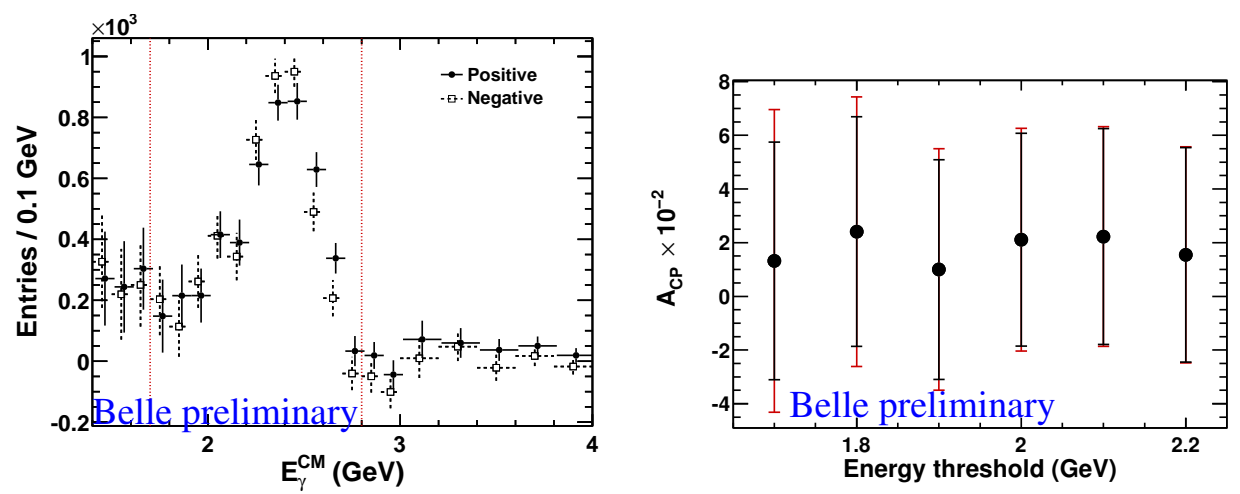

Figure 1: Left: $B \rightarrow X_{s+d} \gamma$ spectrum after background subtraction for positive and negative leptons. Right: measured values of $\mathscr{A}_{C P}$ for different photon energy thresholds. The inner uncertainty bars are statistical and the outer ones include also systematic uncertainties.

\section{3. $B \rightarrow X_{s} \gamma$ branching fraction}

The $B \rightarrow X_{s} \gamma$ branching fraction is theoretically well understood, this decay is dominated by a penguin loop diagram, making it a good candidate to search for new physics. The branching fraction has been calculated up to next-to-next leading order, yielding $\mathscr{B}\left(B \rightarrow X_{s} \gamma\right)=(3.15 \pm$ $0.23) \times 10^{-4}$ for a $E_{\gamma}^{*}>1.6 \mathrm{GeV}$ in the $B$-meson rest frame [5]. The current experimental world average is $\mathscr{B}\left(B \rightarrow X_{s} \gamma\right)=(3.55 \pm 0.24 \pm 0.09) \times 10^{-4}$, consistent with the SM prediction [6].

In the semi-inclusive approach (sum-of-exclusive final states) as many exclusive final states as possible are reconstructed, to reduce the uncertainty from unmeasured modes. The $B$ meson is reconstructed as the combination of a photon with CM energy $E_{\gamma}^{*}=[1.8,3.4] \mathrm{GeV}$ and one of 38 hadronic $X_{s}$ final states. The $X_{s}$ states consist of up to three kaons $\left(K^{+}, K_{S}^{0}\right)$, up to four pions $\left(\pi^{+}, \pi^{0}\right)$ with up to two $\pi^{0}$ and at most one $\eta$ meson. $D$ meson decays are vetoed by reconstructing the $D$ mass. Continuum background is suppressed using a NN constructed with topological variables, the modified Fox-Wolfram moments and $\Delta E$. The selection requirement on it maximizes the statistical significance in the region $M_{X_{s}}=[2.2,2.8] \mathrm{GeV} / \mathrm{c}^{2}$.

The signal is extracted in an unbinned maximum-likelihood fit to $M_{\mathrm{bc}}$ in $19 M_{X_{s}}$ bins. An example is shown in Fig. 2. The dominant systematic uncertainties come from the fragmentation model and the determination of the fit PDFs. The fraction of missing modes is estimated by varying the Pythia parameters that model the $X_{s}$ fragmentation. The branching fraction for $M_{X_{s}}<2.8 \mathrm{GeV} / \mathrm{c}^{2}$ and $E_{\gamma}^{*}>1.8 \mathrm{GeV}$ is $\mathscr{B}\left(B \rightarrow X_{s} \gamma\right)=(3.51 \pm 0.17 \pm 0.33) \times 10^{-4}$, where the first uncertainty is statistical and the second systematic. This result can be extrapolated to $E_{\gamma}^{*}>1.6 \mathrm{GeV}$ for comparison with the theoretical estimate: $\mathscr{B}\left(B \rightarrow X_{s} \gamma\right)=(3.74 \pm 0.18 \pm 0.35) \times 10^{-4}$. This result is in agreement with the SM estimate and previous experimental results [6]. 


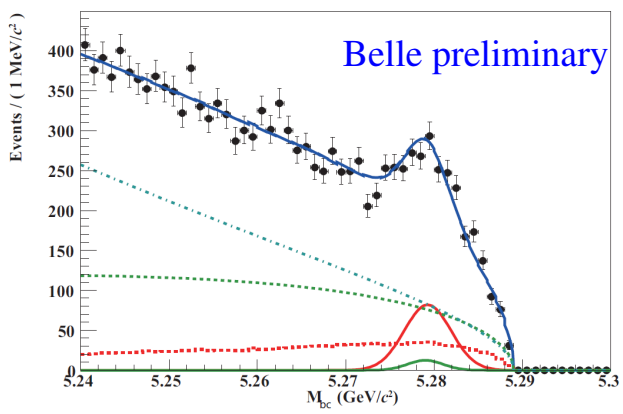

Figure 2: Result of the fit to $M_{\mathrm{bc}}$ in the bin $M_{X_{s}}=[1.9,2.0] \mathrm{GeV} / \mathrm{c}^{2}$ for the $B \rightarrow X_{s} \gamma$ analysis from Belle. The components are: signal (solid red), crossfeed (dashed red), peaking background (solid green), non-peaking $B \bar{B}$ background (dashed green) and continuum (dot-dashed cyan).

\section{Forward-backward asymmetry in $B \rightarrow X_{S} \ell^{+} \ell^{-}$}

The decay $B \rightarrow X_{s} \ell^{+} \ell^{-}$proceeds either via an electroweak penguin loop or a box diagram. The decay amplitude is a function of the Wilson coefficients $C_{7}, C_{9}$ and $\mathrm{C}_{10}$. The forward-backward asymmetry $\left(\mathscr{A}_{\mathrm{FB}}\right)$ is defined as:

$$
\mathscr{A}_{F B}=\frac{\Gamma_{\text {forward }}-\Gamma_{\text {backward }}}{\Gamma_{\text {forward }}+\Gamma_{\text {backward }}},
$$

where $\Gamma$ is the $B \rightarrow X_{s} \ell^{+} \ell^{-}$decay rate. The forward $(\cos \theta>0)$ and backward $(\cos \theta<0)$ regions are defined for the angle $\theta$ between the $\ell^{+}$and the $B$ momentum in the dilepton CM frame. The asymmetry is measured as a function of $q^{2}=M_{\ell^{+} \ell^{-}}^{2}$ since non-SM effects can significantly modify the $q^{2}$ dependence. The asymmetry $\mathscr{A}_{F B}$ has been measured in the decays $B \rightarrow K^{(*)} \ell^{+} \ell^{-}$by various experiments [6]. The present measurement is the first that studies $\mathscr{A}_{\mathrm{FB}}$ as the sum of exclusive final states. This approach provides the most precise comparison to inclusive theoretical predictions.

The $B$ meson is reconstructed from a pair of oppositely charged electrons or muons and one of 10 hadronic $X_{S}$ states. $X_{S}$ is reconstructed as one $K$ or $K_{S}^{0}$ and up to four $\pi$, with at most one $\pi^{0}$. Background from charmonium decays $B \rightarrow X J / \psi(\psi(2 S)), \mathrm{J} / \psi(\psi(2 S)) \rightarrow \ell^{+} \ell^{-}$is rejected by applying a veto which excludes the regions around the $J / \psi$ mass from -400 to $150 \mathrm{MeV} / \mathrm{c}^{2}$ ( -250 to $100 \mathrm{MeV} / \mathrm{c}^{2}$ ) and the $\psi(2 S)$ mass from -250 to $100 \mathrm{MeV} / \mathrm{c}^{2}\left(-150\right.$ to $\left.100 \mathrm{MeV} / \mathrm{c}^{2}\right)$ for electrons (muons). The veto regions for electrons are wider to account for bremsstrahlung photons. To suppress continuum and $B \bar{B}$ background 6 kinematic variables and the modified Fox-Wolfram moments are used to train a NN.

The signal is extracted performing a fit to the $M_{\mathrm{bc}}$ distribution in four $q^{2}$ bins for forward and backward regions. The $q^{2}$ bins are defined as $[0.2,4.3],[4.3,7.3(8.1)],[10.5(10.2), 11.8(12.5)]$, $[14.3,25.0] \mathrm{GeV}^{2} / \mathrm{c}^{2}$ for electrons (muons). The value of the raw asymmetry $\mathscr{A}_{F B}^{\text {raw }}$ deviates from the true value due to the low signal reconstruction efficiency for low $q^{2}$ and high $\cos \theta$. A factor $\alpha$ is determined to correct for this effect. It relates the true and raw asymmetries as $\mathscr{A}_{F B}=\alpha \cdot \mathscr{A}_{F B}^{\mathrm{raw}}$. This correction factor, along with the estimation of peaking background are the dominant systematic uncertainties in this measurement, the statistical uncertainty is, however, dominant. Figure 3 shows the measured value of $\mathscr{A}_{F B}$ as a function of $q^{2}$. The $\mathscr{A}_{F B}$ results are consistent with the SM prediction. The results in the 3 rd and 4 th bin exclude $\mathscr{A}_{F B}<0$ at the $2.3 \sigma$ level [7]. 


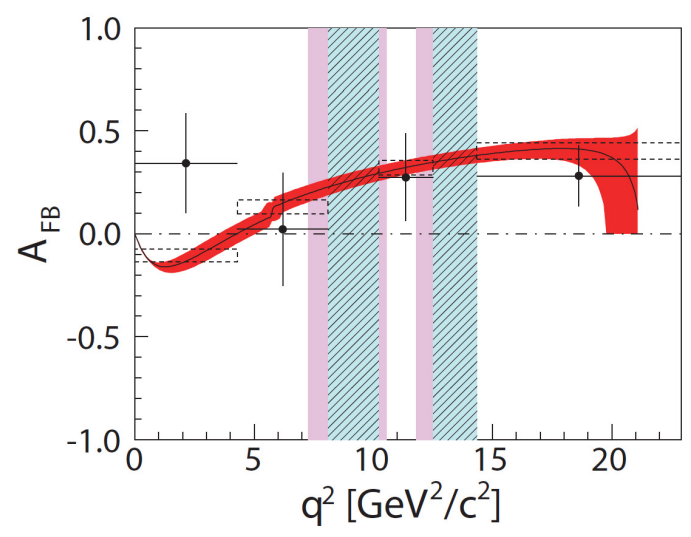

Figure 3: Measured $\mathscr{A}_{F B}$ as a function of $q^{2}$ in $B \rightarrow X_{S} \ell^{+} \ell^{-}$decays. The red band and dashed boxes represent the SM prediction, unbinned and binned respectively. The filled circles show the fit results. The $J / \psi$ and $\psi(2 S)$ vetoes are shown as teal hatched regions. For electrons, the pink shaded regions are added due to the recovery of bremsstrahlung photons.

\section{Search for $B^{0} \rightarrow p \bar{\Lambda} \pi^{-} \gamma$}

Hadronic final states including a $\Lambda$ baryon make up only a small fraction of the $b \rightarrow s \gamma$ final states. The branching fraction $\mathscr{B}\left(B^{+} \rightarrow p \bar{\Lambda} \gamma\right)=\left(2.45_{-0.38}^{+0.44} \pm 0.22\right) \times 10^{-6}$ has been measured by Belle [8]. A hierarchy has been observed in baryonic $B$ decays with $b \rightarrow s$ and $b \rightarrow c$ transitions: $\mathscr{B}\left(B^{+} \rightarrow p \bar{\Lambda} \pi^{+} \pi^{-}\right)>\mathscr{B}\left(B^{0} \rightarrow p \bar{\Lambda} \pi^{-}\right)>\mathscr{B}\left(B^{+} \rightarrow p \bar{\Lambda}\right)$ and $\mathscr{B}\left(B^{0} \rightarrow p \bar{\Lambda}_{c} \pi^{+} \pi^{-}\right)>\mathscr{B}\left(B^{+} \rightarrow\right.$ $\left.p \bar{\Lambda}_{c} \pi^{-}\right)>\mathscr{B}\left(B^{0} \rightarrow p \bar{\Lambda}_{c}\right)$, respectively.

The signal $B$ is reconstructed with a photon with $\mathrm{CM}$ energy $E_{\gamma}^{*}>1.7 \mathrm{GeV}$ and a $\Lambda$ hyperon reconstructed in the decay $\Lambda \rightarrow p \pi$. Due to the rare signature of the event, $B \bar{B}$ background is highly suppressed and continuum is dominant. Continuum suppression uses a likelihood function constructed with the modified Fow-Wolfram moments and $\cos \theta_{B}$, where $\theta_{B}$ is the angle between the $B$ flight direction and the beam direction. The signal is extracted in a 2-dimensional maximumlikelihood fit in the variables $M_{\mathrm{bc}}$ and $\Delta E$. The fit results are shown in Fig. 4.
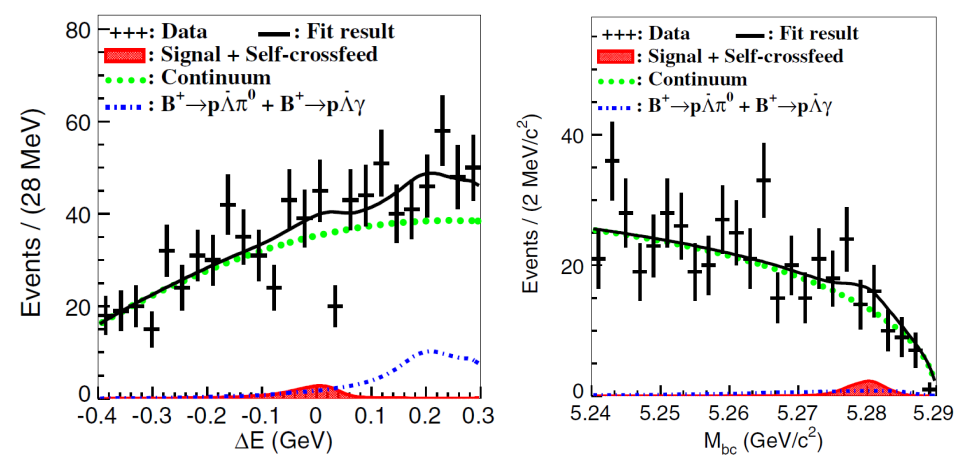

Figure 4: Fit results of the $B^{0} \rightarrow p^{+} \bar{\Lambda} \pi^{-} \gamma$ analysis. Left: $\Delta E$ for $M_{\mathrm{bc}}>5.27 \mathrm{GeV} / \mathrm{c}^{2}$, right: $M_{\mathrm{bc}}$ for $\Delta E<$ $0.05 \mathrm{GeV}$. Shown are the fit result (black line), continuum (green dotted), $B^{+} \rightarrow p^{+} \bar{\Lambda} \pi^{0}$ and $B^{+} \rightarrow p^{+} \bar{\Lambda} \gamma$ (blue dash-dotted) and signal plus self-crossfeed (red). The points with error bars are the Belle data.

The fitted signal yield is $9.5_{-10.7}^{+11.5}$, with a significance of $0.9 \sigma$. An upper limit on the branching 
fraction is determined, which at the $90 \%$ confidence level is $\mathscr{B}\left(B^{+} \rightarrow p \bar{\Lambda} \pi^{-} \gamma\right)<6.5 \times 10^{-7}$ [9]. The hierarchy observed in baryonic $B$ decays is not observed in the radiative decays, as $\mathscr{B}\left(B^{+} \rightarrow\right.$ $\left.p \bar{\Lambda} \pi^{-} \gamma\right)<\mathscr{B}\left(B^{+} \rightarrow p \bar{\Lambda} \gamma\right)$.

\section{Conclusions and outlook}

The transitions $b \rightarrow d \gamma, b \rightarrow s \gamma$ and $b \rightarrow s \ell^{+} \ell^{-}$provide a rich set of observables that can be used to verify SM predictions and search for new physics effects. So far all results show good consistency with the SM. The measurement of the CP asymmetry in $B \rightarrow X_{s+d} \gamma$ decays is the most precise to date. It shows good consistency with the SM and is stable with respect to photon energy threshold in the range $E_{\gamma}^{*}=[1.7,2.2] \mathrm{GeV}$. The latest Belle measurement of $\mathscr{B}\left(B \rightarrow X_{s} \gamma\right)$ is based on the sum of exclusive final states, reconstructing 38 exclusive $X_{s}$ final states. We have also presented the first measurement of the forward-backward asymmetry in $B \rightarrow X_{s} \ell^{+} \ell^{-}$decays analyzed as a sum of exclusive final states. Finally, the search for $B^{0} \rightarrow p \bar{\Lambda} \pi^{-} \gamma$ results in an upper limit in its branching fraction.

In the analyses presented, the statistical uncertainty is an important or even the most important contribution to the total uncertainty. This leaves room for improvement when the large data sets from Belle II become available. Additionally, well established as well as new experimental techniques have been applied, the analyses at future super $B$-factory can greatly profit from them.

\section{References}

[1] A. J. Buras, "Operator product expansion, renormalization group and weak decays," Lect. Notes Phys. 558, 65 (2000) [hep-ph/9901409].

[2] K. Abe et al. [Belle Collaboration], “Evidence for $B^{0} \rightarrow \pi^{0} \pi^{0}$," Phys. Rev. Lett. 91, 261801 (2003) [hep-ex/0308040].

[3] M. Benzke, S. J. Lee, M. Neubert and G. Paz, "Long-Distance Dominance of the CP Asymmetry in $B \rightarrow X_{s, d} \gamma$ Decays,” Phys. Rev. Lett. 106, 141801 (2011) [arXiv:1012.3167 [hep-ph]].

[4] T. Hurth, E. Lunghi and W. Porod, "Untagged $\bar{B} \rightarrow X_{(s+d)} \gamma C P$ asymmetry as a probe for new physics," Nucl. Phys. B 704 (2005) 56 [hep-ph/0312260].

[5] M. Misiak and M. Steinhauser, "NNLO QCD corrections to the $\bar{B} \rightarrow X_{s} \gamma$ matrix elements using interpolation in $m_{c}$," Nucl. Phys. B 764, 62 (2007) [hep-ph/0609241].

[6] Y. Amhis et al. [Heavy Flavor Averaging Group Collaboration], "Averages of B-Hadron, C-Hadron, and tau-lepton properties as of early 2012,” arXiv:1207.1158 [hep-ex].

[7] Y. Sato et al. [Belle Collaboration], "Measurement of the Lepton Forward-Backward Asymmetry in Inclusive $B \rightarrow X_{s} \ell^{+} \ell^{-}$Decays," arXiv:1402.7134 [hep-ex].

[8] M. -Z. Wang et al. [Belle Collaboration], "Study of $B^{+} \rightarrow$ LLambdar, pLambda $\pi^{0}$ and $B^{0} \rightarrow$ LLambda $\pi^{-}$,” Phys. Rev. D 76, 052004 (2007) [arXiv:0704.2672 [hep-ex]].

[9] Y. T. Lai et al. [Belle Collaboration], “Search for $B^{0}$ topLambda $\pi^{-} \gamma$ at Belle,” arXiv:1312.4228 [hep-ex]. 